\title{
Development of a Novel Two-Hand Playing Piano Robot
}

\author{
Chin-Shyurng Fahn, Cheng-Feng Tsai, Yung-Wei Lin \\ Department of Computer Science and Information Engineering, National Taiwan University of Science and Technology \\ No. 43, Keelung Rd., Sec. 4, Da'an Dist., Taipei City 10607, Taiwan (R. O. C.) \\ csfahn@mail.ntust.edu.tw; D10415002@mail.ntust.edu.tw; M9715038@mail.ntust.edu.tw
}

\begin{abstract}
Currently, we realize a two-hand playing piano robot which can recognize printed piano score in real time and then play piano by itself. The playing piano robot is composed of two subsystems: a vision system and a two-hand playing system. In this paper, we focus on presenting the two-hand playing system that consists of a finger system and an arm system for each hand. For the finger system, we design a simple signal control module which can receive a signal from the host computer and drive an exact tubular solenoid after the signal is decoded. The arm system is driven by two linear motors, each of which carries a hand to run a different gamut by sliding from left to right or vice versa. In addition, because the sliding ranges of these two linear motors are overlapped, we create a collision avoidance mechanism to ensure their motions are safe and sound. The experimental results reveal that the two-hand playing system can play theme and accompaniment simultaneously which has the ability of playing chords. The safe speed of playing must be smaller than tempo 100. So far, the two-hand playing system works smoothly and undeniably.
\end{abstract}

Keywords: two-hand playing piano robot, chord playing, arm motion collision avoidance, linear motor, tubular solenoid.

\section{Introduction}

In the past, the researches and developments of robots stressed the applications of industry. They usually employed robots to accomplish duplicated or dangerous work. There were few robots active in the human life. Recently, the situation has altered considerably, because the habits of the human life are changing. In the near future, many developed countries will face a problem of population aging. In addition, the technology of semiconductor has been improved greatly. The computing ability of computers is becoming more and more powerful, and the size of computers is becoming small. It is worth mentioning that the ability of power storage of batteries attains a big breakthrough, say the lithium battery. Consequently, many novel robotic applications have been published by academia and industrials, and their achievements are growing rapidly and continuously. For instance, the clearing robot can do a clean job in indoors environments [1], the entertainment robot can play a flute by itself [2], and the guiding robot can lead guests to the location we assign [3].

To take a broad view in the future, the human being will depend on high technical products gradually owing to the convenience of using them in daily life. The cost of manufacturing robots will reduce, because both the hardware and software used for developing robots are more and more ordinary. We believe that the researches of robotic applications will become popular and the development of robotic industry is ripe or not will affect the competitiveness between countries. Nowadays, the general robotic applications are still mainly in the industrial field. People do not feel much the effect on robots that are applied to the human life. In order to let people have chances to understand the advantage of applying robots to improve the human life, many companies had developed entertainment robots that were already published. The Partner robot is one of the well-known robots, which was made by the Toyota Motor Company [4]. This robot acts like the human being, which can play trumpet or violin, and moves itself on the stage. The Pleo robot was made by the Ugobe Company [5], which has the appearance of a dinosaur. There are many sensors equipped on the body, so it can interactive with people.

In this paper, we aim to develop an intelligent robot which can recognize piano score and play piano in real time. We expect this robot behaves more like human being; that is, not getting commands passively from users. The robot can automatically determine whether the object in front of a PTZ camera is piano score or not, and play the music information of the piano score by itself. 


\section{System Description}

The system architecture as well as the system flowchart of our developed experimental robot is described in this section. Both the arm controlling system and the finger controlling system inclusive of the specifications of some crucial constituting parts are also elaborated.

\subsection{The Hardware System}

The developed experimental robot is $120 \mathrm{~cm}$ long, $60 \mathrm{~cm}$ wide, and $105 \mathrm{~cm}$ high.It consists of two main subsystems: a vision system and a two-hand playing system. The vision system comprises one PTZ camera and one video grabber. The two-hand playing system is made up of two linear motors, two AC motor drivers, thirty-two tubular solenoids, and two finger controllers.Figure 1 illustrates the system architecture of the experimental robot, which is composed of three layers. We put the PTZ camera at the top layer, the two linear motors at the medium layer, and one PC as well as AC motor drivers together with finger controllers at the lowest layer. The concrete experimental robot is demonstrated in Figure 2.

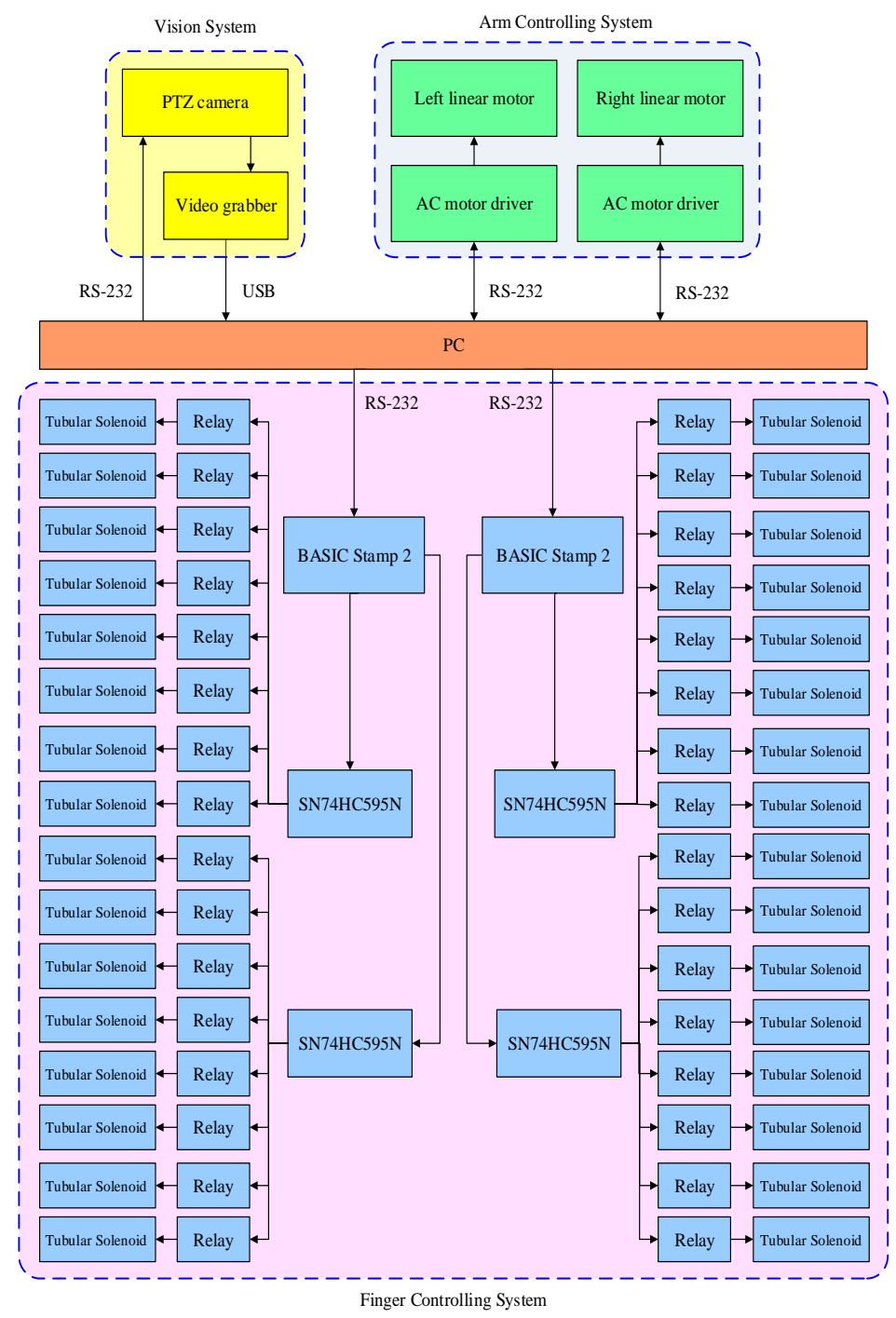

Fig. 1: The system architecture of our developed experimental robot. 


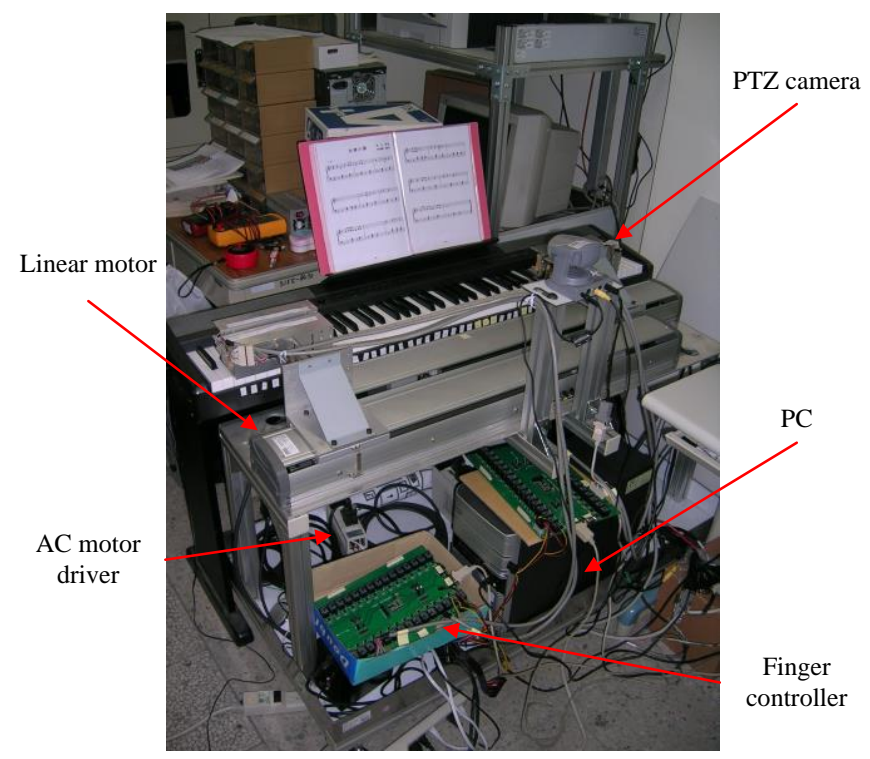

Fig. 2: The concrete experimental robot.

In order to fetch the image of printed piano score, we employ a PTZ camera. This camera can pan and tilt to stare at the target and room in to get a clearer image. After fetching the image, we acquire the information of music notes by means of image processing and pattern recognition techniques. According to this, two linear motors are instructed to move to a proper position and press down a correct piano key. Figure 3shows the system flowchart of our experimental robot.

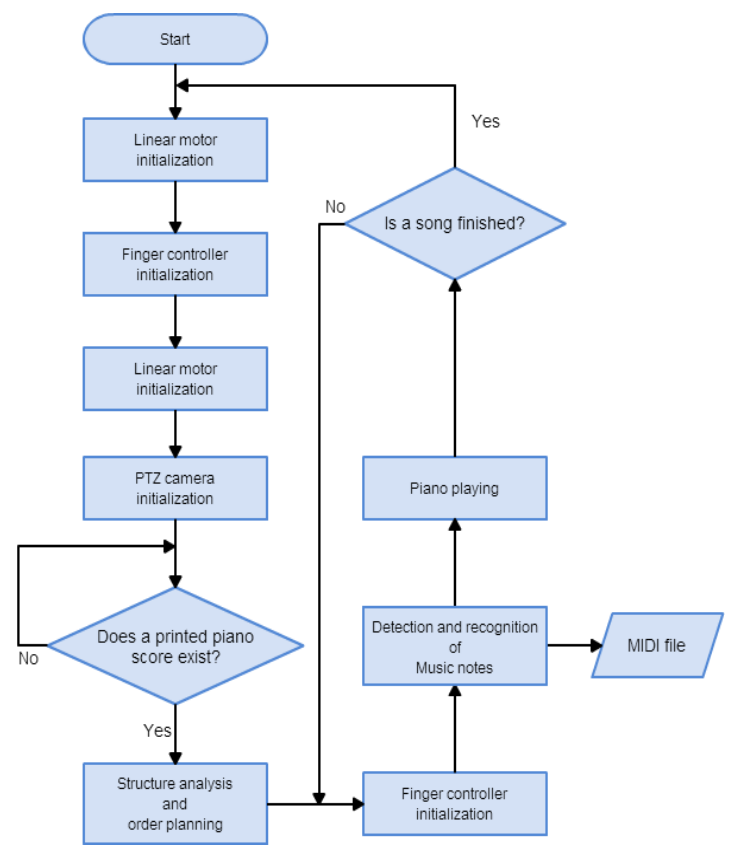

Fig. 3: The system flowchart of the experimental robot.

\subsection{The Arm Controlling System}

1) Specification of the linear motor

In order to simulate the action of people playing piano, we equip two linear motors on the robot as shown in Figure 4. The linear motor is generally installed in the factory for making or carrying products, which possesses high accuracy. Table lexpresses the specification of the linear motor. 


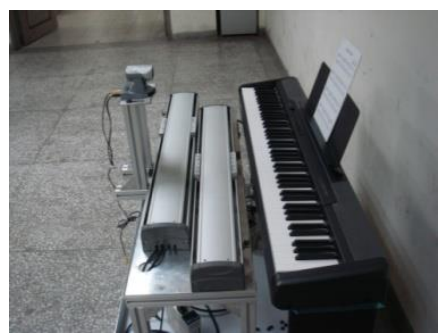

Fig. 4: Two linear motors adopted to imitate human arms.

Table 1: The Specification of the Linear Motor.

\begin{tabular}{|c|c|}
\hline Specification & Value \\
\hline Move range & $500 \mathrm{~mm}$ \\
\hline Length & $1030 \mathrm{~mm}$ \\
\hline Weight & $17.9 \mathrm{~kg}$ \\
\hline Motor type & Mitsubishi $200 \mathrm{~W}$ \\
\hline
\end{tabular}

2) Specification of the AC motor driver

The linear motor's motion is controlled by a motor driver. We choose the motor driver MR-J2S-20A1 produced by Mitsubishi as shown in Figure 5. And Table 2 is the specification of the AC motor driver.

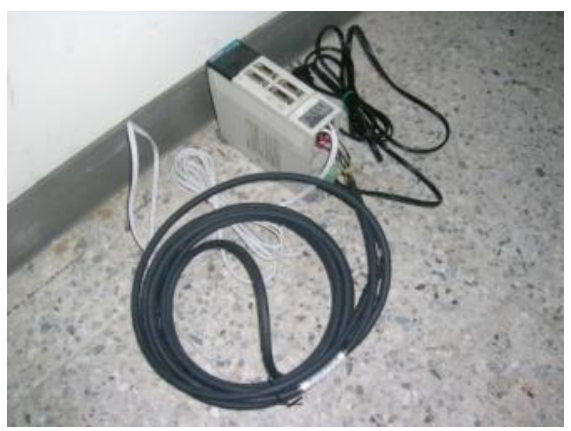

Fig. 5: The AC motor driver used for controlling the linear motor.

Table 2: The Specification of the AC Motor Driver.

\begin{tabular}{|c|c|}
\hline Specification & Value \\
\hline Voltage & 1-phase 100V to 120V AC \\
\hline Rated output & 200W \\
\hline System & Sine-wave PWM control, current control system \\
\hline Dynamic brake & $0 \sim 55^{\circ} \mathrm{C}$ \\
\hline Control mode & Position mode, Speed mode and Torque mode \\
\hline Ambient temperature & $90 \% \mathrm{RH}$ or less (non-condensing) \\
\hline Ambient humidity & $0.7 \mathrm{~kg}$ \\
\hline Weight & \\
\hline
\end{tabular}

3) Instruction and controlling of the AC motor driver

We correctly move the linear motor to press piano keys in different compasses. Before moving, we must know the present position and calculate the distance between the desired position and the present position. Finally, we control the linear motor to move to the correct position by virtue of Forward, Backward and Stop commands. Table 3 lists the frequent used instructions of the AC motor driver. 
Table 3: The Frequent Used Instructions of the AC Motor Driver.

\begin{tabular}{|c|c|c|}
\hline Function & Command & Remark \\
\hline Forward & A018h + distance & Distance is positive \\
\hline Backward & A0138h + distance & Distance is negative \\
\hline Stop & A0154h+1EA5 & Get pulse \\
\hline Present position & 018B0h+NULL & Get revolution \\
\hline Present speed & 018C0h+NULL & Range is between $0 \sim 5175$ \\
\hline Set speed & A0104h+speed & Range is between $0 \sim 20000$ \\
\hline Set acceleration & A0118h+acceleration &
\end{tabular}

\subsection{The Finger Controlling System}

1) Specification of the tubular solenoid

In order to simplify the playing mechanism, we adopt the tubular solenoid RG-T-1632Sas shown in Figure 6 produced by the Richmeg Industry Company to simulate human fingers to play piano keys. Table 4 describes the specification of the tubular solenoid that is at a stage fixed on the linear motor.If two piano keys arelocated apart over five keys and must be pressed at the same time, thenwe cannot play at this situation. For solving this problem, one hand is created to be equipped with sixteen tubular solenoids as Figure 7 demonstrates. The distance between the leftmost and the rightmost tubular solenoids is just one octave

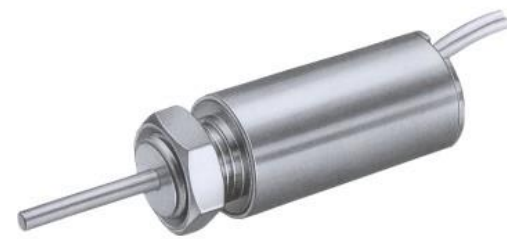

Fig. 6: A concrete tubular solenoid.

Table 4: The Specification of the Tubular Solenoid.

\begin{tabular}{|c|c|c|}
\hline Specification & Value & Remark \\
\hline Length & $41.53 \mathrm{~mm}$ & \\
\hline Diameter & $16 \mathrm{~mm}$ & \\
\hline Weight & $47 \mathrm{~g}$ & \\
\hline Duty cycle & $25 \%$ & $\begin{array}{c}\text { MAX. "on" time in } \\
\text { seconds }\end{array}$ \\
\hline Voltage DC & $12 \mathrm{~V}$ & \\
\hline
\end{tabular}

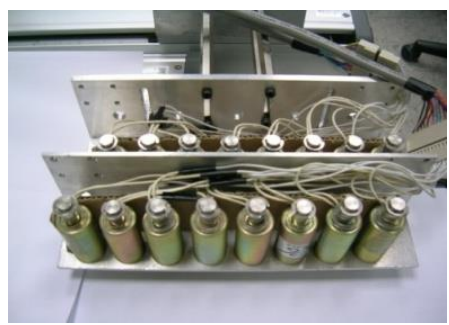

Fig. 7: A robot's hand equipped with sixteen tubular solenoids.

2) The structure of the finger controller

There are thirty two tubular solenoids used in our two-hand playing system. In order to turn on/off these tubular solenoids, we design a simple control circuitas shown in Figure 8. First, we send a command to the microprocessor Basic Stamp 2 (BS2) via a transmission line RS-232,and the BS2 will distribute the command to a proper chip SN74HC595. Finally, the chip will decode the command and drive the correct relay. 


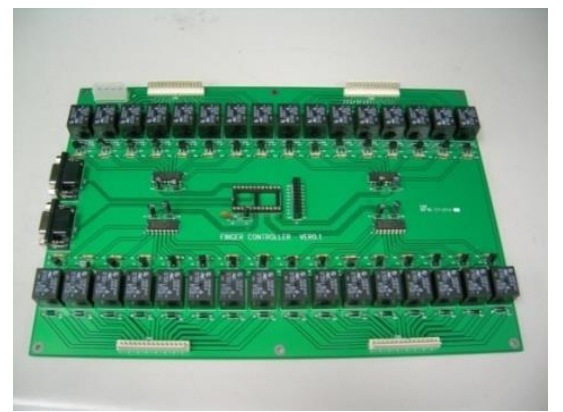

Fig. 8: The control circuit used for turning on/off tubular solenoids.

3) The instruction of the finger controller

Each hand has sixteen tubular solenoids in the finger controlling system. For the convenience ofdescription, these tubular solenoids are named as Figure 9 shows.

Because different relays are driven by four chips SN74HC595, we name these chips are Left_f, Left_r, Right_f, and Right_r. The tubular solenoids from L1 to L8 are controlled by Left_f, from L9 to L16 are controlled by Left_r, from R1 to R8 are controlled by Right_f and from R9 to R16 are controlled by Right_r, respectively. Table 5 depicts the codes of all the tubular solenoids.

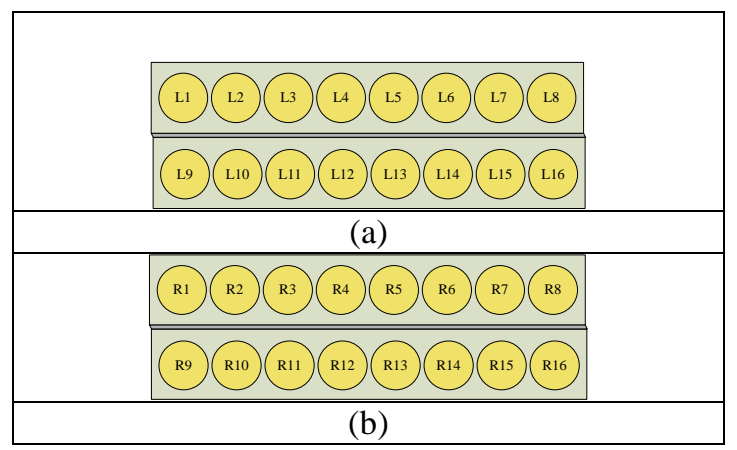

Fig. 9: The name of each tubular solenoid in: (a) the left hand; (b) the right hand.

Table 5: The Codes of All the Used Tubular Solenoids.

\begin{tabular}{|c|c|c|c|c|c|c|c|}
\hline $\begin{array}{c}\text { Tubular } \\
\text { solenoid }\end{array}$ & Left_f & $\begin{array}{c}\text { Tubular } \\
\text { solenoid }\end{array}$ & Right_f & $\begin{array}{c}\text { Tubular } \\
\text { solenoid }\end{array}$ & Left_r & $\begin{array}{c}\text { Tubular } \\
\text { solenoid }\end{array}$ & Right_r \\
\hline L1 & 0X01 & R1 & 0X01 & L9 & 0X01 & R9 & 0X01 \\
\hline L2 & 0X02 & R2 & 0X02 & L10 & 0X02 & R10 & 0X02 \\
\hline L3 & 0X04 & R3 & 0X04 & L11 & 0X04 & R11 & 0X04 \\
\hline L4 & 0X08 & R4 & 0X08 & L12 & 0X08 & R12 & 0X08 \\
\hline L5 & 0X10 & R5 & 0X10 & L13 & 0X10 & R13 & 0X10 \\
\hline L6 & 0X20 & R6 & 0X20 & L14 & 0X20 & R14 & 0X20 \\
\hline L7 & 0X40 & R7 & 0X40 & L15 & 0X40 & R15 & 0X40 \\
\hline L8 & 0X80 & R8 & 0X80 & L16 & 0X80 & R16 & 0X80 \\
\hline
\end{tabular}

\section{The Two-hand Playing System}

This section presents the design essence of the two-hand playing system, including piano keys positioning techniques and the manner of playing piano in detail. Besides, the collision avoidance mechanism for the two arms as well as the playing and encoding of the chord are emphatically depicted.

\subsection{Piano Keys Positioning and the Manner of Playing}

In this development, we aim to build a two-hand playing piano robot that can recognize printed piano score like human being. In order to reach the purpose of playing, we install two linear motors, thirty two tubular solenoids, and one 
digital piano. There are eighty eight keys on the piano that can be played from Pitch A1 to Pitch C9. The movement unit of the linear motor is by "pulse", and the distance $1 \mathrm{~mm}$ is about equal to 6,429 pulses by measurement. For keys positioning, we set the tubular solenoidno. 9 be the reference point. By calculation, the distance between thecenters of two concatenated keys is $24 \mathrm{~mm}$ that isrequired about 154,082 pulses as illustrated in Figure10.Therefore, if we want to move the linear motor to the keyk $\mathrm{k}_{\mathrm{d}}$,we just need to count how many keys $(\mathrm{N})$ between $\mathrm{k}_{\mathrm{d}}$ and the present position. Subsequently, we obtainthe movement argument from $\mathrm{N}$ multiplied by 154,082.

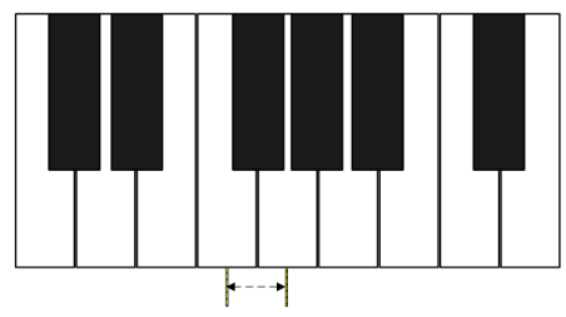

The distance is $24 \mathrm{~mm}$ required

Fig. 10: The distance between the centres of two concatenated keys.

On the part of playing, we expect to store the recognition result of piano score in MIDI format. This format stores a number twice, which is used to stand for a pitch. The first number means "playing" and the second number means "stopping". In this manner, the time interval between the appearancesof the first number and the second number means the length of playing.For cooperating the MIDI format, we deploy an array of 128 elementsto record which tubular solenoids are pressed or not.

\subsection{Collision Avoidance for the Arm}

We apply two linear motors to simulate the action of arms while people play piano. For extending the range as wide as possible, we let two linear motorsslide widely between the left and right ends under the limits of mechanism. The left linear motor can move between Pitch C2 and Pitch C5, and the right one can move between Pitch C4 and Pitch C7as shown in Figure 11.

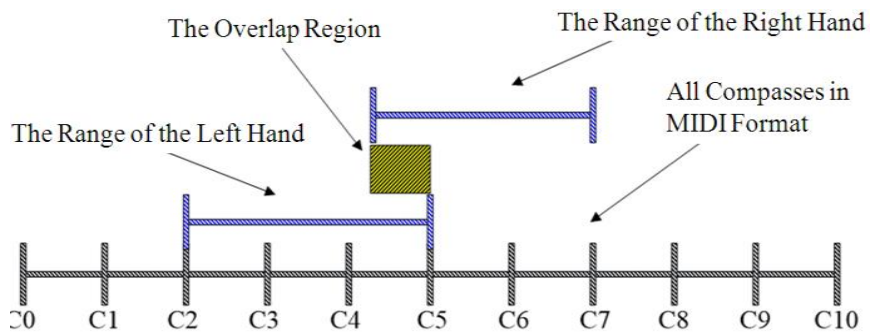

Fig. 11: The sliding ranges of the two linear motors.

Because some moving ranges of the two linear motorsare overlapped, we design a measure to prevent them collided with each other. The shortest length between tubular solenoids L9 and R9 is 9 widths of a key. Hence, we do the following checks whenever a linear motor is moved. Take the left linear motor as an example. 


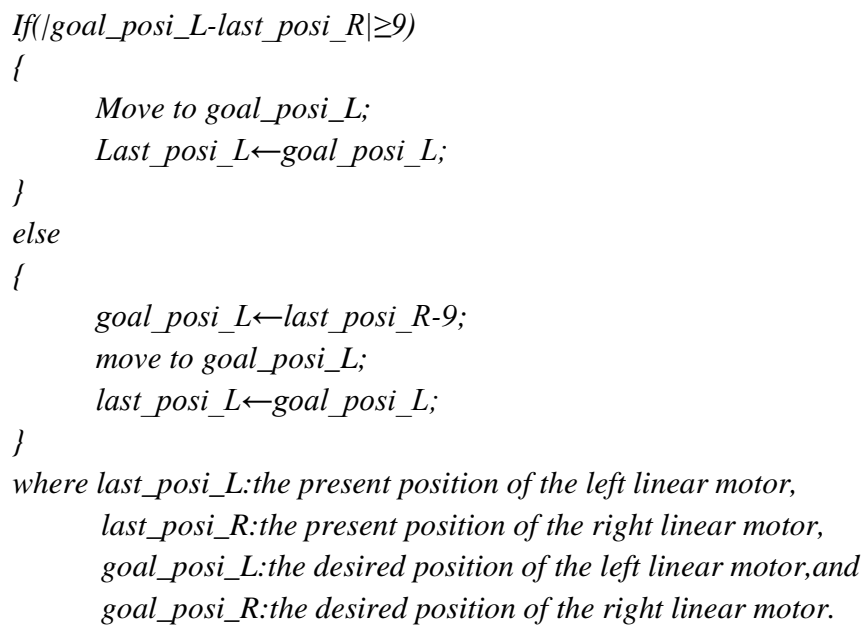

\subsection{Playing and Encoding of the Chord}

In the two-hand playing system, we construct a finger controller consisting of micro processor BS2 and IC 74595 to control tubular solenoids. However, the BS2 cannot carry out more than one instruction meanwhile. In order to play chords, we must drive many tubular solenoids by virtue of encoding at the identical time. The following example shows how to encode the driven sequence of the tubular solenoids. Because the tubular solenoid no. L9 may stay in the positions of Pitches C, D, E, F, G, A, and B, we can produce seven models according to these positions.

Suppose the tubular solenoid no. L9 stay in the position of Pitch C now. The system needs to press Keys E5, G\#5, and B5 simultaneously. These three pitches in MIDI format are 64, 68, and 71, respectively. There are twelve semitones in a compass, so we get remainders 4,8 , and 11 using the above values divided by 12 . In advance, we edit seven tables for easily finding the instruction of a tubular solenoid corresponding to a certain pitch. From these tables, we can acquire the instructions of the related tubular solenoids 0x04, 0x10, and 0x40 individually. At last, we obtain the follow encoding result by the logical operator OR. An example of using the left linear motor is given below.

$$
\begin{aligned}
& \text { left_F }=0 \times 10 \\
& l \text { left_ } R=0 \times 04 \mid 0 \times 40=0 \times 44
\end{aligned}
$$

\section{Experimental Results and Discussions}

Many experiments are made to evaluate the effectiveness of the two-hand playing system of our developed playing piano robot. In the experiments, it is seen that the playing system works undeniably.

\subsection{The Installation of the Experimental Equipments}

First, we put printed piano score on the stand of piano, and then move the robot to the position of performance. We make the tubular solenoid L9 aim at the piano key C2. And the distance between the centres of the PTZ camera and the piano score is $55 \mathrm{~cm}$ as Figure 12 shows. 


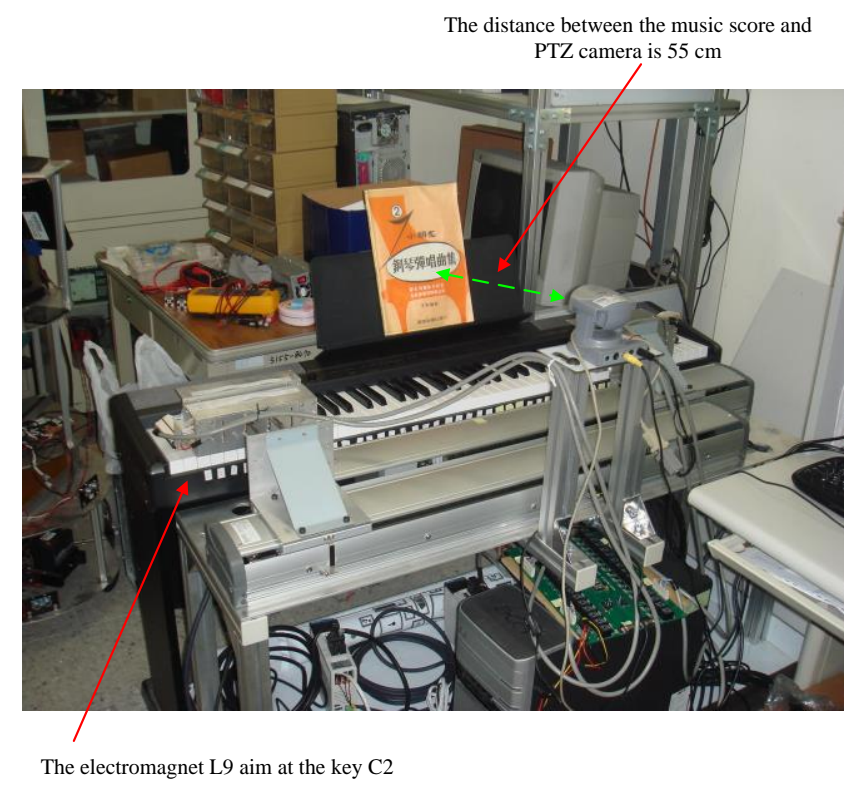

Fig. 12: The installation of the experimental equipments.

Next, we get the parameter of the start position of robot's arm by executing the correct procedure and the corresponding piano key acquired from the parameteris send to the robot. Finally, we check all communication lines whether have been plugged in or not, and then start the robot. Table 6 shows the specifications of the host computer and software that we used.

Table 6: The Specifications of the Used Host Computer and Software.

\begin{tabular}{|c|l|}
\hline \multicolumn{1}{|c|}{ Hardware } & \multicolumn{1}{c|}{ Software } \\
\hline & Operating system: \\
& Microsoft XPProfessional SP3 \\
& Developing tools: \\
& Borland C++ Builder 6.0 \\
Host computer: & BASIC Stamp Editor Software \\
Intel Pentium D 3.0 GHz with 1 GB RAM CPU & Music score editor: \\
& Overture 4.0 \\
& Music player: \\
& Microsoft Windows Media Player 9 \\
\hline
\end{tabular}

\subsection{The Operation of the Two-hand Playing System}

The main procedure of our playing system manipulates the operation of the two-hand playing piano robot. It will transform the pitches of the piano score and voice lengths into the commands of controlling the playing piano robot by encoding. Once the playing system receives the commands, it will move robot's arm to a correct position. In order to ensure that robot's arm is moved to the correct position, the playing system will determine whether the position is correct or not relying on the feedback capability of the linear motor. After pressing a piano key, a spring is employed to restore the axle of the tubular solenoid. Prior to complete restoration, the axle is prevented from hitting the piano key. It is required that the linear motor must release all the tubular solenoids and wait $100 \mathrm{~ms}$ before moving. From the experimental results, the safe speed of playing must be smaller than tempo 100 .

For easy orientating, we mainly play piano by the tubular solenoids L9 and R9 when performing. But, if a chord will be played, we will join the other tubular solenoids. Additionally, the two-hand playing system can avoid the unexpected collision of the two linear motors. The chord in a song can make melody plentiful. However, it will make the playing system become complicated. For solving the problem of chord playing, the tubular solenoids L9 and R9 are selected to be the reference. We establish seven tables based on the seven positions C, D, E, F, G, A and B that the tubular solenoids L9 
and R9 may stay. These tables record the corresponding commands of different pitches. According to the test results, the chord can be played successfully.

\subsection{The Recognition Result Stored in MIDI format}

In the future, we want to edit and manage the recognition result of printed piano score and do other application by some software. For storing the recognition result, the well-known musical format "MIDI" is adopted. In order to verify the validity of the MIDI file we edit, both the software Overture and Windows Media Player are applied to check the editing result.

The Overture is one of the famous musical editing software which can output the MIDI file based on the editing score or transform the MIDI file into a score image. In the light of the experimental results, our playing system can transform the MIDI file into the score image correctly. And the Windows Media Player is a celebrated multimedia player which supports many formats of music. Of course, it also includes the MIDI format. In order to test our edited MIDI file, we play the file using the Windows Media Player. The experimental results reveal that our system can play the MIDI file smoothly.

\section{Conclusion}

In the two-hand playing system, we hope that its mechanism is simple but the function is without loss of completeness. We implement the two-hand playing system mainly using two linear motors and thirty-two tubular solenoids, because the operation of the tubular solenoid is easy and the accuracy of the linear motor is good. The actual manipulation is effortless and very reliable. From the experimental results, we see that the two-hand playing system can press correct piano keys easily by means of the position of piano keys together with the MIDI format we design. Besides this, our created collision avoidance mechanism achieves valid actions. There occur no collisions when the two-hand playing system is working. Finally, owing to the limit of hardware, we have to play chord by a command through a coding method. The chord melody can be played in one octave simultaneously and without errors in many experiments we test.

Although there is a great difference between the appearances of the two-hand playing system and real human upper limbs, the system we design has good integrity in the aspect of functions. To compare with the playing system composed of multidimensional robot arms and fingers, our two-hand playing system has the following advantages: low cost as well as easy control and maintenance. It is effective to surmount the shortcomings of existing playing piano robots.

\section{Acknowledgements}

The authors thank the National Science Council of Taiwan (R. O. C.) for supporting this work in part under Grant NSC-101-2221-E-011-143-MY3.

\section{References}

[1] iRobot: Clean Robots, [Online]. Available: http://store.irobot.com/shop/index.jsp?categoryId=2804605

[2] K. Chida et al, "Development of a new anthropomorphic flutist robot WF-4," in Proceedings ofthe IEEE International Conference on Robotics and Automation, Barcelona, Spain, vol. 1, pp. 152-157, 2004.

[3] Y. Sakagami et al, "The intelligent ASIMO: System overview and integration," in Proceedings ofthe IEEE/RSJ International Conference on Intelligent Robots and Systems, Lausanne, Switzerland, vol. 3, pp. 2478-2483, 2002.

[4] Y. Kusuda, "Toyota's violin-playing robot," International Journal of Industrial Robot, vol. 35, no. 6, pp. 504-506, 2008.

[5] PLEO robots, [Online]. Available: http://www.ucube.com.tw/page4.html 